TRANSACTIONS OF THE

AMERICAN MATHEMATICAL SOCIETY

Volume 359, Number 1, January 2007, Pages 227-247

S 0002-9947(06)03892-X

Article electronically published on August 15, 2006

\title{
REDUCING AND ANNULAR DEHN FILLINGS
}

\author{
SANGYOP LEE
}

\begin{abstract}
If two Dehn fillings on a simple manifold create a reducible manifold and an annular manifold respectively, then the distance between those filling slopes is known to be at most two. Moreover, Eudave-Muñoz and Wu gave infinitely many examples of manifolds admitting reducing and annular Dehn fillings at distance two. In this paper, we complement their examples to establish a complete list of simple manifolds admitting such a pair of Dehn fillings.
\end{abstract}

\section{INTRODUCTION}

Consider surfaces of non-negative Euler characteristic, i.e., sphere, disk, torus or annulus. We say that such a surface is small [6], and that a compact, connected, orientable 3-manifold is simple if it contains no essential small surfaces [26. Small surfaces have played an important role in our understanding of the topology of 3-manifolds. For example, the prime decomposition due to Kneser [17 and Milnor 21, or JSJ-decomposition due to Jaco and Shalen [15] and Johannson 16. Thurston's Geometrization Theorem for Haken manifolds 23 also asserts that a 3 -manifold with non-empty boundary is hyperbolic if and only if it is simple.

Let $M$ be a 3-manifold with a torus boundary component $\partial_{0} M$ and $\rho$ a slope (the isotopy class of an essential circle) on $\partial_{0} M$. We define $M(\rho)$ to be the manifold obtained by $\rho$-Dehn filling, that is, by attaching a solid torus to $M$ along $\partial_{0} M$ so that $\rho$ bounds a disk in the solid torus. This operation has become one of the fundamental methods of constructing 3-manifolds. In particular, it is the wellknown theorem of Lickorish 20] and Wallace 24] that every closed orientable 3manifold can be obtained by a sequence of Dehn fillings on the exterior of a link in $S^{3}$.

Ever since Thurston 23 announced that if $M$ is hyperbolic, then all but finitely many Dehn fillings on $M$ yield hyperbolic manifolds, a great deal of attention has been directed towards obtaining a more precise quantification of this statement. As a part of this project, considerable energy has been devoted to finding how many Dehn filling slopes on a simple manifold yield non-simple ones. More precisely, given a simple manifold $M$, if $\pi$ and $\gamma$ are two slopes on $\partial_{0} M$ such that both $M(\pi)$ and $M(\gamma)$ fail to be simple, then there are ten possible pairs of essential small surfaces that arise, and the best possible upper bounds on the distance $\Delta(\pi, \gamma)$ (the geometric intersection number of $\pi$ and $\gamma$ ) in various cases have been established. See [6] for more detail.

Received by the editors October 27, 2003 and, in revised form, October 20, 2004.

2000 Mathematics Subject Classification. Primary 57N10.

Key words and phrases. Reducible, annular, Dehn filling.

(C)2006 American Mathematical Society Reverts to public domain 28 years from publication 
Furthermore, in some choices of the pair of the involved small surfaces, there are results examining the examples of 3-manifolds which occur at or near the maximal distance. For example, Gordon [5] showed that if both $M(\pi)$ and $M(\gamma)$ contain essential tori, then $\Delta(\pi, \gamma) \leq 5$ except for four specific manifolds realizing $\Delta=$ $6,7,8$, and Gordon and $\mathrm{Wu}$ [12, 14] showed that if $M(\pi)$ contains an essential annulus and $M(\gamma)$ contains an essential torus (or annulus), then $\Delta(\pi, \gamma) \leq 3$ except for three specific manifolds realizing $\Delta=4,5$. Recently, Lee 18 also showed that there is exactly one simple manifold $M$ admitting a pair of Dehn fillings $M(\pi)$, $M(\gamma)$, each of which contains an essential sphere and a Klein bottle, with $\Delta(\pi, \gamma)=$ 3 , the maximal distance in that case.

In this paper, we are interested in the case that $M(\pi)$ and $M(\gamma)$ contain an essential sphere and an essential annulus, respectively. In this case, $\mathrm{Wu}$ [26] and independently Qiu 22] proved that $\Delta(\pi, \gamma) \leq 2$. Moreover, Wu also showed that if we put an additional homological condition $H_{2}\left(M, \partial M-\partial_{0} M\right) \neq 0$, then $\Delta(\pi, \gamma) \leq$ 1. In particular, this condition holds if $M$ either has a boundary component with genus at least 2 , or if it has more than two boundary tori. Thus when $\Delta(\pi, \gamma)=2$, $\partial M$ must be a union of two tori. On the other hand, Eudave-Muñoz and $\mathrm{Wu} 3$. Theorem 2.6] constructed infinitely many examples of simple manifolds admitting reducing and annular Dehn fillings at distance 2. However, their examples are not all simple manifolds with such a pair of Dehn fillings. In the present paper, we will give a complete list of such manifolds.

Theorem 1.1. If $M$ is a simple manifold such that $M(\pi)$ is reducible and $M(\gamma)$ is annular with $\Delta(\pi, \gamma)=2$, then $M$ is one of the manifolds that appears in Theorem 2.1 .

In the case of $\Delta(\pi, \gamma)=1$ not much is known; it is not known, for example, which surfaces can be the boundary of $M$, or if there is an upper bound for the number of times an essential annulus may hit the attached solid torus.

The structure of the paper is as follows: In Section 2 we extend Eudave-Muñoz and Wu's construction to find an infinite family of simple manifolds with reducing and annular Dehn fillings at distance 2. The rest of the paper is devoted to showing that the family is composed of all such simple manifolds. Before we start proving Theorem 1.1. we make a few preliminary observations in Sections 3 and 4, which are about properties of curves on surfaces and checkerboard colored graphs on the 2-sphere. As usual, the assumption that $M(\pi)$ and $M(\gamma)$ each contains an essential sphere and an essential annulus with $\Delta(\pi, \gamma)=2$ leads to two labelled graphs in the 2-sphere and the annulus. A result of [19] allows us to assume that the graph in the annulus has exactly two vertices. In Section 5 we collect general lemmas about the two labelled graphs. Section 6 is the main section of the paper, in which it is shown that the graph in the 2 -sphere contains $S$-cycles. We then prove Theorem 1.1 in Section 7

The author would like to thank the referee for his/her helpful comments and corrections to the original text.

\section{Dehn fillings CREATing ESSEntial SPHeRES AND ANnUli}

In this section, we slightly extend Eudave-Muñoz and Wu's method in [3, Section 2] to give a list of simple manifolds admitting reducing and annular Dehn fillings at distance 2 . 


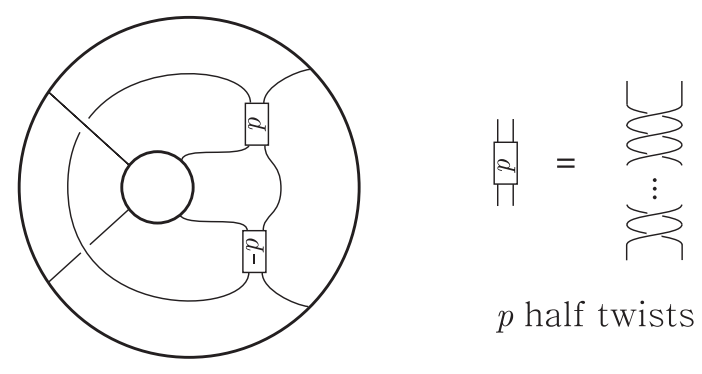

FiguRE 1.

Following [3, Figure 2.1], let $Y=S^{2} \times I$ and $\xi_{p}$ the tangle in $Y$ as shown in Figure 1. We remark that Eudave-Muñoz and Wu considered only the case that $p$ is even. Let $M_{p}$ be the double cover of $Y$ branched along the tangle $\xi_{p}$. Then $M_{p}$ is a compact 3-manifold with $\partial M_{p}$ a union of two tori. For $r=\infty, 0,-1,-1 / 2$, let $\xi_{p}(r)$ be the tangles in [3, Figure 2.2(a)-(d)] with $2 p$ replaced by $p$, and $M_{p}(r)$ the corresponding double branched covering.

Denote by $C(r, s)$ the cable space of type $(r, s)$ and by $Q(r, s)$ the Seifert fibered space with orbifold a disk with two cone points of indices $r$ and $s$.

Theorem 2.1. The manifolds $M_{p}, p \geq 3$, are mutually distinct simple manifolds, each having two slopes $\pi$ and $\gamma$ at distance 2 such that $M_{p}(\pi)$ is a reducible manifold, the connected sum of a solid torus and the real projective space, and $M_{p}(\gamma)$ is an annular manifold, the union of $C(2,1)$ and $Q(p,-p)$ along their boundary tori.

Proof. We assume familiarity with [3, Section 2]. The argument in [3, Section 2] goes through verbatim in our present context except [3. Lemma 2.5] in the case $p=3$. Thus, we only need to check that $M=M_{3}$ is atoroidal. Let $T_{0}$ and $T_{1}$ be two boundary tori of $M$, where $T_{0}$ is the lift of the inside boundary sphere of $Y$. Using [3, Lemma 2.2], we have the following.

(1) $M(\infty)$ is the connected sum of a solid torus and the real projective space;

(2) $M(0)=Q(2,-4)$;

(3) $M(-1)=Q(4,-2)$;

(4) $M(-1 / 2)$ is the union of $C(2,1)$ and $Q(3,-3)$ with a unique essential torus.

We follow the proof of [3, Lemma 2.5]. Assume that $M$ has an essential torus $T$. Then $T$ must be separating, otherwise $M(\infty)$ would contain a non-separating torus, a contradiction. As in the proof of [3. Lemma 2.5], we may assume that $T$ cuts $M$ into two components $W, W^{\prime}$ so that $W$ contains $T_{0}$ and $W^{\prime}$ contains no essential torus.

Claim. $T$ is compressible in $M(-1 / 2)$.

Proof. $M(-1 / 2)$ has a unique essential torus $T^{\prime}$. So if $T$ is incompressible in $M(-1 / 2)$, then either it is boundary parallel or it is isotopic to $T^{\prime}$. In the first case, $M(-1 / 2)=W(-1 / 2) \cup W^{\prime}=(T \times I) \cup W^{\prime} \cong W^{\prime}$, so $T^{\prime}$ would be an essential torus in $W^{\prime}$, contradicting the choice of $T$. Hence $T$ is isotopic to $T^{\prime}$ in $M(-1 / 2)$ and then $W^{\prime}=C(2,1)$ or $Q(3,-3)$.

First, assume that $W^{\prime}=Q(3,-3)$. Then $W$ contains both $T_{0}$ and $T_{1}$. Since $M(0)$ is atoroidal, $T$ is either boundary parallel or compressible in $M(0)$. In the first case, $W(0)$ is the boundary parallelism, so $Q(2,-4)=M(0)=W(0) \cup W^{\prime} \cong$ 
$W^{\prime}=Q(3,-3)$, a contradiction. In the second case, $T$ is compressible in $W(0)$ and the resulting sphere becomes a reducing sphere in $M(0)$, since it separates the two tori $T$ and $T_{1}$. This is impossible.

Next, assume that $W^{\prime}=C(2,1)$. Then $T_{1} \subset W^{\prime}$. It follows from the argument below [3, Figure 2.3] that there is a homeomorphism of $M$ interchanging the two boundary tori $T_{0}$ and $T_{1}$. Let $W^{\prime \prime}$ be the image of $W^{\prime}$ under this homeomorphism. Let $r$ be the cabling slope on $T_{0} \subset W^{\prime \prime}=C(2,1)$. Then $M(r)$ is reducible, so $\Delta(r, \infty) \leq 1$ by [10, Theorem 1.2]. (Although $M$ is bounded by two tori, the arguments in [10] apply here without change once we note that $M$ is irreducible.) If $\Delta(r,-1 / 2) \geq 2$, then $W^{\prime \prime}(-1 / 2)=Q(2, s)$ with $|s| \geq 2$ and $\partial W^{\prime \prime}(-1 / 2)$ would be the unique essential torus in $M(-1 / 2)$, which is impossible. Thus, $\Delta(r,-1 / 2)=1$ and hence $\Delta(r, \infty)=1$. Solving these equalities, we have $r=0$ or -1 . However, both $M(0)$ and $M(-1)$ are irreducible, a contradiction.

Then the last paragraph of the proof of [3, Lemma 2.5] remains valid here to show that $M$ is atoroidal.

\section{Curves on surfaces}

The rest of the present paper is devoted to the proof of Theorem 1.1. Before starting the proof, we first consider properties of curves on surfaces. We say that a properly embedded arc or a circle in a surface is inessential if it is boundary parallel or null-homotopic in the surface, otherwise it is essential.

Lemma 3.1. Let $F$ be an orientable surface. Let $a$ and $c$ be an oriented arc and an oriented circle in $F$ which intersect transversally. If $c$ intersects a coherently in at least two points, then $c$ is non-separating in $F$.

Proof. Take a subarc $a_{1}$ of $a$ between two consecutive intersection points of $a$ and $c$ in $a$. Since $c$ intersects $a$ coherently, the ends of $a_{1}$ locally lie on different sides. Let $A$ be a thin annulus neighborhood of $c$ in $F$ such that $a_{1}$ intersects $\partial A$ in two points, $p$ and $q$. Then $p$ and $q$ lie on different components of $\partial A$. There is an essential arc $a_{2}$ in $A$ such that $a_{2} \cap c$ is one point and $\partial a_{2}=\{p, q\}$. Then $\left(a_{1}-\operatorname{Int} A\right) \cup a_{2}$ is a circle in $F$ that meets $c$ transversely in one point.

Lemma 3.2. Let $Q$ be a twice-punctured annulus with $\partial_{1} Q$ and $\partial_{2} Q$ the boundaries of the punctures, and let $A$ be an annulus with $\partial A=\partial_{1} Q \cup \partial_{2} Q$. Suppose that $c$ is a separating essential circle in $F=Q \cup A$ such that $c \not \subset Q, c \not \subset A$, and each component of $c \cap Q$ (resp. $c \cap A)$ is an essential arc in $Q$ (resp. A). Then $c \cap Q$ consists of arcs $c_{1}, c_{2}, \ldots, c_{2 n}$ in $Q$ (successively labelled along $c$ ) with the property that

(i) $\partial c_{n} \subset \partial_{1} Q, \partial c_{2 n} \subset \partial_{2} Q$; and

(ii) there is a set of disjoint rectangles $\left\{R_{1}, R_{2}, \ldots, R_{n-1}\right\}$ on $Q$ such that $R_{j}$ realizes the parallelism between $c_{j}$ and $c_{2 n-j}$.

Proof. The surface $F$ is a twice-punctured torus. Here, the boundary components of $\partial Q$ other than $\partial_{1} Q$ and $\partial_{2} Q$ are viewed as the punctures. The curve $c$ cuts off a twice-punctured disk $D$ from $F$ so that $A \cap D$ is a union of properly embedded bands in $D$. These bands must be all parallel in $D$, otherwise some component of $c \cap Q$ would be boundary parallel in $Q$ through a subdisk $E$ in $D$ as shown in Figure 2 (a) where the two small circles indicate the boundary components of $Q$ other than 


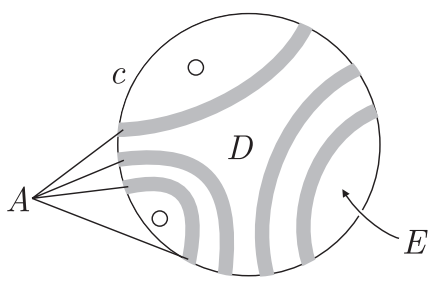

(a)

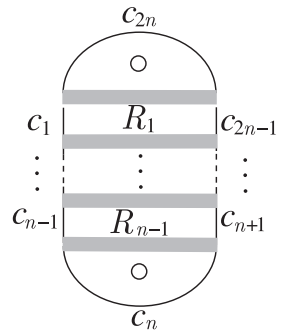

(b)

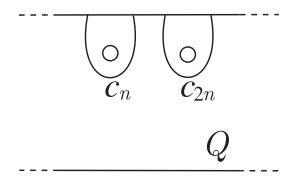

(c)

Figure 2.

$\partial_{1} Q$ and $\partial_{2} Q$. Thus, $A \cap D$ looks as in Figure 2(b). Let $c_{1}, c_{2}, \ldots, c_{2 n}$ be the arcs as indicated in Figure 2(b). Then the property (ii) is obviously satisfied.

We may assume $\partial c_{n} \subset \partial_{1} Q$. Since each component of $c \cap A$ is an essential arc in $A, c \cap \partial_{1} Q$ and $c \cap \partial_{2} Q$ have the same number of intersection points. Thus, if $\partial c_{2 n} \subset \partial_{1} Q$, then some component of $c \cap Q$ would be parallel to a subarc on $\partial_{2} Q$, a contradiction. See Figure 2(c). Thus the property (i) is satisfied.

Let $i_{1}, i_{2}, \ldots, i_{r}(r \leq n)$ be distinct elements of $\{1,2, \ldots, n\}$. Let $\left(i_{1} i_{2} \cdots i_{r}\right)$ denote the permutation that maps $i_{j} \mapsto i_{j+1}$ modulo $r, j=1,2, \ldots, r$, and maps every other element of $\{1,2, \ldots, n\}$ onto itself. Then $\left(i_{1} i_{2} \cdots i_{r}\right)$ is called a cycle of length $r$.

Lemma 3.3. Given two integers $m, n(0<m<n)$, let $\rho:\{1,2, \ldots, n\} \rightarrow$ $\{1,2, \ldots, n\}$ be a permutation defined by $\rho(i)=m+i$ for $1 \leq i \leq n-m$ and $\rho(i)=n-i+1$ for $n-m+1 \leq i \leq n$. If $\rho$ is a cycle of length $n$, then $m=1$ or 2. Moreover, when $m=2, n$ is even.

Proof. First assume $m \leq n / 2$. The diagram in Figure 3(a) indicates a Klein bottle, and the oriented arcs on the rectangle are put together into a set $\mathcal{C}$ of oriented simple closed curves. The numbers on the top and left sides of the rectangle, respectively, match with those on the bottom and right sides via the identification of the sides of the rectangle. Understanding the former numbers as the domain of $\rho$ and the latter numbers as the codomain, we can associate the permutation $\rho$ with this Klein bottle diagram. Notice that $\rho$ is a cycle of length $n$ if and only if $\mathcal{C}$ has a single element.

Now consider the double covering of this diagram, which is a torus, indicated in Figure 3(b), with a set of oriented simple closed curves $\widetilde{\mathcal{C}}$ lifted from $\mathcal{C}$. A horizontal line and a vertical line in the diagram indicate simple closed curves, say, $\lambda$ and $\mu$. The algebraic intersection number of $\widetilde{\mathcal{C}}$ with $\lambda$ is zero and that with $\mu$ is $m$. Thus, $\widetilde{\mathcal{C}}$ can be isotoped into $m$ copies of $\lambda$. Since $\mathcal{C}$ has a single element, $\widetilde{\mathcal{C}}$ has one or two elements, so $m=1$ or 2 . The argument for the case $m>n / 2$ is very similar.

Assume $m=2$. If $n$ is odd, then $\rho$ preserves the parity. Thus, $n$ must be even. 


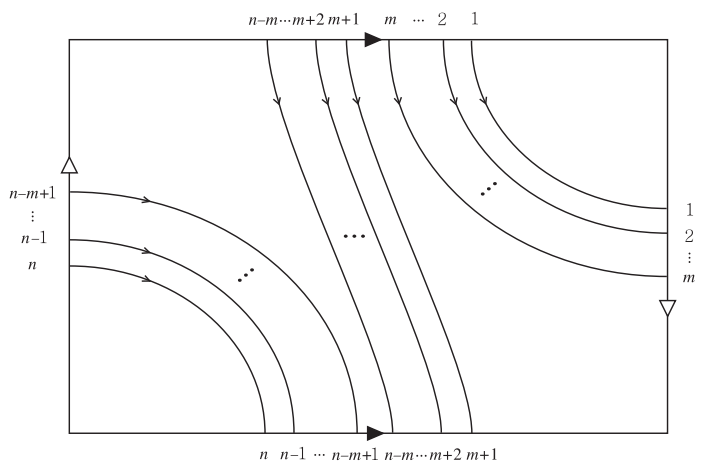

(a)

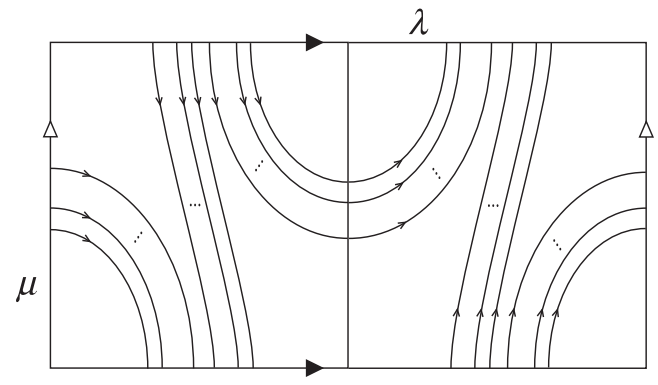

(b)

FiguRE 3.

\section{GRAPHS ON THE 2-SPHERE}

In this section we prepare Proposition 4.2, which will be used in Section 6 to show that the annular Dehn filled manifold contains a properly embedded Möbius band.

Lemma 4.1. Let $\Lambda$ be a graph on $S^{2}$ such that every vertex of $\Lambda$ has valence 4 . Then $\Lambda$ contains a disk face with at most 3 sides.

Proof. Let $V, E$ and $F$ be the numbers of vertices, edges and disk faces of $\Lambda$, respectively. Then $2 E=4 V$, giving $E=2 V$. Applying Euler characteristic formula for $\Lambda$, we obtain $V-E+F \geq \chi$ (sphere) $=2$ and hence $F \geq V+2$. Now suppose that every disk face of $\Lambda$ has at least 4 sides. Then $4 V+8 \leq 4 F \leq 2 E=4 V$, a contradiction.

Let $\Lambda$ be a directed graph on $S^{2}$. If a disk face of $\Lambda$ is bounded by a circuit of consistently oriented edges, we call it a cycle face.

Suppose that each edge of $\Lambda$ is labelled either $\alpha$ or $\beta$ and that each face is colored either black or white. Then travelling around a disk face of $\Lambda$ gives a cyclic sequence of signed labels. For example, if one goes for the orientation of an $\alpha$-labelled edge, then it contributes $\alpha^{+}$to the cyclic sequence, and if one goes against, then it contributes $\alpha^{-}$. Note that if a face of $\Lambda$ is a cycle face, then only one of the signs, + and - , appears in the associated cyclic sequence. We shall say that two disk faces of $\Lambda$ of the same color are isomorphic if one can obtain the same cyclic sequences 


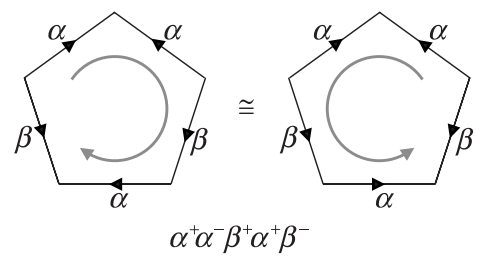

FiguRe 4.

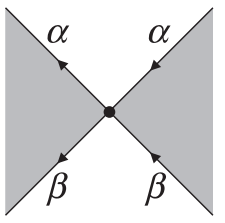

$r$

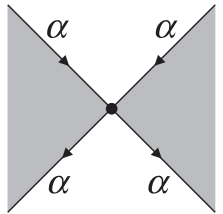

$S$

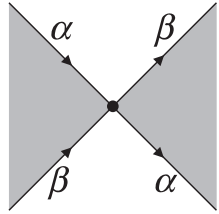

$t$

FiguRE 5.

of signed labels by reading around their boundaries in some directions. See Figure 4.

We remark that every face of $\Lambda$ is a disk if and only if $\Lambda$ is connected.

Proposition 4.2. Let $\Gamma$ be a connected directed graph on $S^{2}$ such that the faces are checkerboard colored with black and white colors, the edges are labelled $\alpha$ or $\beta$, and the vertices each have valence 4 so that the local view of $\Gamma$ at each vertex looks like one of the diagrams in Figure 5. Suppose that $\Gamma$ has a single isomorphic class $\mathcal{B}$ of black faces and two isomorphic classes $\mathcal{A}$ and $\mathcal{C}$ of white faces and that every face in $\mathcal{A}$ is not a cycle face and has at least 4 sides. Then one of the following holds:

(1) $\Gamma$ contains no vertex of the second type in Figure 5 .

(2) every face in $\mathcal{B}$ is a cycle face bounded only by $\alpha$-labelled edges; or

(3) every face in $\mathcal{C}$ is a cycle face bounded either only by $\alpha$-labelled edges or only by $\beta$-labelled edges. Moreover, its edges come only from the first diagram in Figure 5 .

Proof. Let $r, s, t$ be the numbers of vertices of $\Gamma$ near which the local view of $\Gamma$ looks like the first, second or third diagram in Figure 5, respectively. Assume $s>0$.

If a face in $\mathcal{B}$ is a cycle face, then from the local view of $\Gamma$, one can see that it is bounded only by $\alpha$-labelled edges. Thus, from now on, we assume that any black face is not a cycle face. Then both labels $\alpha$ and $\beta$ appear on the sides of each black face. Now it is enough to show that the assertion (3) holds. The second sentence in the assertion (3) clearly follows from the first sentence and Figure 5 .

The graph $\Gamma$ has neither a sink nor a source, so $\Gamma$ must have cycle faces. Thus, the faces in $\mathcal{C}$ are cycle faces. We also assume that both labels $\alpha$ and $\beta$ appear on the sides of each face in $\mathcal{C}$ and eventually we get a contradiction.

Let $V, E$ and $F$ be the numbers of vertices, edges and faces of $\Gamma$, respectively. Then we have $V=r+s+t$ and $2 E=4 V$, giving $E=2(r+s+t)$. An Euler characteristic calculation gives $V-E+F=\chi($ sphere $)=2$ and hence $F=r+s+t+2$. 
Let $F_{\mathcal{A}}, F_{\mathcal{B}}$ and $F_{\mathcal{C}}$ be the numbers of faces in classes $\mathcal{A}, \mathcal{B}$ and $\mathcal{C}$. Then $F_{\mathcal{C}}>0$ because $\Gamma$ has cycle faces. Since $F=F_{\mathcal{A}}+F_{\mathcal{B}}+F_{\mathcal{C}}$, we have

$$
F_{\mathcal{A}}+F_{\mathcal{B}}+F_{\mathcal{C}}=r+s+t+2 .
$$

Let $X_{\mathcal{A}}$ be the number of corners of a face in $\mathcal{A}$ at which an $\alpha$-labelled edge and a $\beta$-labelled edge meet, and $Y_{\mathcal{A}}$ (resp. $Z_{\mathcal{A}}$ ) the number of corners of a face in $\mathcal{A}$ at which two $\alpha$-labelled (resp. $\beta$-labelled) edges meet. Similarly for $X_{\mathcal{B}}, Y_{\mathcal{B}}, Z_{\mathcal{B}}, X_{\mathcal{C}}$, $Y_{\mathcal{C}}, Z_{\mathcal{C}}$. Then $X_{\mathcal{A}}, X_{\mathcal{B}}, X_{\mathcal{C}}$ are even integers and $X_{\mathcal{B}}, X_{\mathcal{C}} \geq 2$ by our assumption. Also, for example, $X_{\mathcal{B}}+Y_{\mathcal{B}}+Z_{\mathcal{B}}$ is the number of sides of a black face. In fact, $Z_{\mathcal{B}}=0$. (See Figure 5.)

The black corners between two $\alpha$-labelled edges occur only at the vertices of $\Gamma$ in the second diagram in Figure 5, so we have

$$
Y_{\mathcal{B}} F_{\mathcal{B}}=2 s
$$

and $Y_{\mathcal{B}}>0$, since $s>0$.

Similarly, by counting white corners between an $\alpha$-labelled edge and a $\beta$-labelled edge, we have

$$
X_{\mathcal{A}} F_{\mathcal{A}}+X_{\mathcal{C}} F_{\mathcal{C}}=2 t .
$$

Claim. $Y_{\mathcal{B}}=1$.

Proof. If $X_{\mathcal{A}}>0$ (and then $X_{\mathcal{A}} \geq 2$ ), then $2\left(F_{\mathcal{A}}+F_{\mathcal{C}}\right) \leq X_{\mathcal{A}} F_{\mathcal{A}}+X_{\mathcal{C}} F_{\mathcal{C}}=2 t$, giving $F_{\mathcal{A}}+F_{\mathcal{C}} \leq t$ and then combining with the equations (4.1) and (4.2), we have $s<r+s+2 \leq F_{\mathcal{B}}=2 s / Y_{\mathcal{B}}$. Hence $Y_{\mathcal{B}}=1$.

Assume $X_{\mathcal{A}}=0$. Then every face in $\mathcal{A}$ is bounded only by $\alpha$-labelled edges or only by $\beta$-labelled edges. If the latter holds, then from Figure 5, one can see that the faces in $\mathcal{A}$ are cycle faces. Thus, every face in $\mathcal{A}$ is bounded only by $\alpha$-labelled edges.

Suppose a face (and hence every face) in $\mathcal{A}$ has two consecutive edges whose orientations are consistent. Then every face in $\mathcal{A}$ contains a corner such as the white corner between two $\alpha$-labelled edges in the first diagram of Figure 5 . This implies $F_{\mathcal{A}} \leq r$. Since $X_{\mathcal{A}}=0$, from the equation (4.3) we have $X_{\mathcal{C}} F_{\mathcal{C}}=2 t$, giving $F_{\mathcal{C}} \leq t$. These two inequalities and the equation (4.1) give $s+2 \leq F_{\mathcal{B}}=2 s / Y_{\mathcal{B}}$. Hence $Y_{\mathcal{B}}=1$.

Suppose a face (and hence every face) in $\mathcal{A}$ does not have such a pair of edges, that is, every two consecutive edges of the face have the opposite orientations. Then the white corners in the first diagram of Figure 5 belong to the faces in $\mathcal{C}$, meaning $Y_{\mathcal{C}} F_{\mathcal{C}}=Z_{\mathcal{C}} F_{\mathcal{C}}=r$.

If $r>0$, then $Y_{\mathcal{C}}, Z_{\mathcal{C}} \geq 1$. Combining with $X_{\mathcal{C}} \geq 2$, we have $X_{\mathcal{C}}+Y_{\mathcal{C}}+Z_{\mathcal{C}} \geq 4$, i.e., every face in $\mathcal{C}$ has at least 4 sides. Since every face in $\mathcal{A}$ has also at least 4 sides, Lemma 4.1 guarantees that every black face has at most 3 sides. That is, $X_{\mathcal{B}}+Y_{\mathcal{B}}+Z_{\mathcal{B}} \leq 3$, and hence the inequalities $X_{\mathcal{B}} \geq 2$ and $Y_{\mathcal{B}}>0$ give $Y_{\mathcal{B}}=1$.

Assume $r=0$. Then $Y_{\mathcal{C}}=Z_{\mathcal{C}}=0$. If $X_{\mathcal{C}} \geq 4$, then the above argument shows $Y_{\mathcal{B}}=1$. Hence we may assume $X_{\mathcal{C}}=2$, that is, every face in $\mathcal{C}$ is a bigon bounded by an $\alpha$-labelled edge and a $\beta$-labelled edge. Then two white regions in the third diagram of Figure 5 represent faces in $\mathcal{C}$, and two white regions in the second diagram represent faces in $\mathcal{A}$. This means that the faces in $\mathcal{A}$ and the faces in $\mathcal{C}$ cannot be adjacent. Since $\Gamma$ is connected, either $s=0$ or $t=0$. Both are absurd, since we assumed $s>0$ and we observed $F_{\mathcal{C}}>0$. 
For any white face $f$ of $\Gamma$, if an edge $e$ in $\partial f$ is $\beta$-labelled, then the next and the previous edges in $\partial f$ have the same orientation as $e$. See Figure 5 , Thus, one can see that if $f$ belongs to class $\mathcal{A}$, then $f$ has at least two pairs of consecutive $\alpha$ labelled edges with opposite orientations, since $f$ is not a cycle face. Then $F_{\mathcal{A}} \leq s$. By the above claim and the equation (4.2), we have $F_{\mathcal{B}}=2 s$. So,

$$
F_{\mathcal{A}} \leq F_{\mathcal{B}} / 2 \text {. }
$$

The equality $2(r+s+t)=E=\left(X_{\mathcal{B}}+Y_{\mathcal{B}}+Z_{\mathcal{B}}\right) F_{\mathcal{B}}=\left(X_{\mathcal{B}}+1\right) F_{\mathcal{B}}$ gives

$$
r+s+t=\left(X_{\mathcal{B}}+1\right) F_{\mathcal{B}} / 2 .
$$

From (4.1), (4.4), 4.5), we have $F_{\mathcal{C}} \geq\left(X_{\mathcal{B}} / 2-1\right) F_{\mathcal{B}}+2$.

Let $\left|\alpha_{\mathcal{C}}\right|$ be the number of $\alpha$-labelled edges belonging to the faces in $\mathcal{C}$. By our assumption, every face in $\mathcal{C}$ has at least one $\alpha$-labelled edge of $\Gamma$, so we have

$$
\left|\alpha_{\mathcal{C}}\right| \geq F_{\mathcal{C}} \geq\left(X_{\mathcal{B}} / 2-1\right) F_{\mathcal{B}}+2 .
$$

On the other hand, the number of $\alpha$-labelled edges of each black face is $X_{\mathcal{B}} / 2+Y_{\mathcal{B}}$, so the number of $\alpha$-labelled edges of $\Gamma$ is $\left(X_{\mathcal{B}} / 2+1\right) F_{\mathcal{B}}$. The four edges incident to the vertex in the second diagram in Figure 5 do not belong to a face in $\mathcal{C}$, since every face in $\mathcal{C}$ is a cycle face. This means

$$
\left|\alpha_{\mathcal{C}}\right| \leq\left(X_{\mathcal{B}} / 2+1\right) F_{\mathcal{B}}-4 s=\left(X_{\mathcal{B}} / 2-1\right) F_{\mathcal{B}},
$$

since $F_{\mathcal{B}}=2 s$. The two inequalities conflict, a contradiction proving the proposition.

\section{The GRAPHS OF INTERSECTION}

From now on, we will assume that $M$ is a simple 3 -manifold whose boundary consists of two tori, $\partial_{0} M$ and $\partial_{1} M$. Let $\pi$ and $\gamma$ be two slopes on $\partial_{0} M$ such that $M(\pi)$ is reducible and $M(\gamma)$ is annular with $\Delta(\pi, \gamma)=2$.

Let $\widehat{P}$ be a reducing sphere in $M(\pi)$ which intersects the attached solid torus $V_{\pi}$ in a family of meridian disks. We assume that $\widehat{P}$ is chosen so that $p=\left|\widehat{P} \cap V_{\pi}\right|$ is minimal over all such reducing spheres in $M(\pi)$. Then $P=\widehat{P} \cap M$ is an essential planar surface in $M$, with $p$ boundary components, each having slope $\pi$ on $\partial_{0} M$. Since $M$ is simple, we have $p \geq 3$.

Similarly, let $\widehat{Q}$ be either an essential annulus or a properly embedded Möbius band in $M(\gamma)$ which intersects the attached solid torus $V_{\gamma}$ in a family of meridian disks so that $Q=\widehat{Q} \cap M$ has a minimal number of boundary components over all such surfaces. Let $q=\left|\widehat{Q} \cap V_{\gamma}\right|$. Each component of $\partial Q \cap \partial_{0} M$ has slope $\gamma$. Then we have the following lemma which is crucial throughout the rest of this paper.

Lemma 5.1. $q=1$ or 2 according to whether $\widehat{Q}$ is a Möbius band or an annulus.

Proof. This is [19, Theorem 1.2].

Lemma 5.2. Both $P$ and $Q$ are incompressible and boundary incompressible in $M$.

Proof. This follows from the minimality of $p$ and $q$.

By a standard argument we may assume that $P$ meets $Q$ transversely, in properly embedded arcs and circles such that no arc component of $P \cap Q$ is boundary parallel in either $P$ or $Q$, and no circle component bounds a disk in either $P$ or $Q$. We 

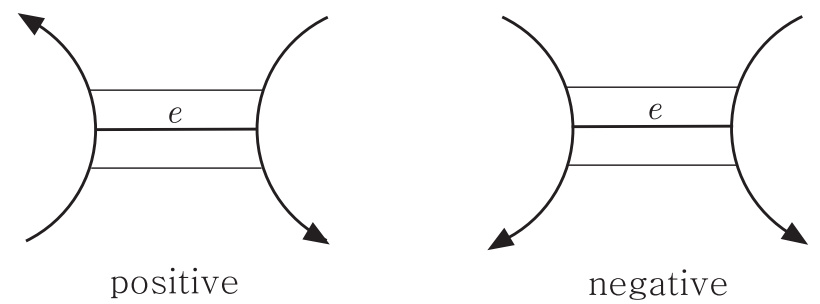

FIGURE 6.

also assume that each component of $\partial P$ meets each component of $\partial Q \cap \partial_{0} M$ in $\Delta(\pi, \gamma)=2$ points.

Let $G_{P}$ be the graph in $\widehat{P}$ with the disks of $\widehat{P} \cap V_{\pi}$ as (fat) vertices, and the arc components of $P \cap Q$ as edges. Similarly for $G_{Q}$. Let $u_{1}, \ldots, u_{p}$ (resp. $v_{1}, \ldots, v_{q}$ ) be the disks of $\widehat{P} \cap V_{\pi}$ (resp. $\widehat{Q} \cap V_{\gamma}$ ), labelled successively when travelling along the attached solid torus. This gives a numbering of the vertices of $G_{P}$ (resp. $G_{Q}$ ). Furthermore, it induces a labelling of the edge-endpoints in $G_{P}$ and $G_{Q}$ in such a way that if an edge-endpoint $x$ in $G_{P}$ or $G_{Q}$ is the intersection of $\partial u_{i}$ and $\partial v_{j}$, then $x$ is labelled $j$ in $G_{P}$, while it is labelled $i$ in $G_{Q}$. The labels of the edge-endpoints in $G_{P}$ (resp. $G_{Q}$ ) are considered as integers modulo $q$ (resp. $p$ ). Then, for example, the labels in $G_{Q}$, when going around $\partial v_{j}$, appear as $1,2, \ldots, p$ repeated $\Delta$ times.

Although $Q$ may be non-orientable, one can establish a parity rule, which is a generalization of the usual one. First, orient all components of $\partial P$ ( or $\partial Q \cap \partial_{0} M$ ) so that they are mutually coherent on $\partial_{0} M$. Let $e$ be an edge in $G_{P}$ (or $\left.G_{Q}\right)$. Then $e$ has a disk neighborhood $D$ in $P$ (or $Q$ ) with $\partial D=a \cup b \cup c \cup d$, where $a$ and $c$ are arcs in $\partial P$ (or $\left.\partial Q \cap \partial_{0} M\right)$ with induced orientations from $\partial P$ (or $\partial Q$ ). On $D$, if $a$ and $c$ have opposite directions, then $e$ is called positive, otherwise negative. See Figure 6. Then we have the following.

Parity rule 5.3. An edge is positive on one graph if and only if it is negative on the other graph.

Let $G$ denote $G_{P}$ or $G_{Q}$. An edge in $G$ is said to be level if its endpoints have the same label. An $x$-edge is an edge in $G$ with label $x$ at one endpoint. A cycle in $G$ is a subgraph which becomes an embedded circle after shrinking fat vertices on it to points. An $x$-cycle is a cycle of positive $x$-edges which can be oriented so that the tail of each edge has label $x$. A Scharlemann cycle is an $x$-cycle that bounds a disk face of $G$. Each edge of a Scharlemann cycle has the same label pair. A Scharlemann cycle of length two is called an $S$-cycle in short. A set of four parallel positive edges is called an extended Scharlemann cycle if the two middle edges form an $S$-cycle. Let $G^{+}$denote the subgraph of $G$ consisting of all vertices and all positive edges of $G$. The reduced graph $\bar{G}$ is defined to be the graph obtained from $G$ by amalgamating each complete set of parallel edges into a single edge, and for an edge $\alpha$ of $\bar{G}$, let $w(\alpha)$, the weight of $\alpha$, denote the number of edges of $G$ in $\alpha$. We can give a sign to each edge in $\bar{G}$ according to the sign of the members in it.

Lemma 5.4. (1) $G_{Q}$ cannot have a positive level edge.

(2) $G_{Q}$ cannot have an extended Scharlemann cycle.

(3) Any two Scharlemann cycles of $G_{Q}$ have the same label pair. 
(4) Suppose $\widehat{Q}$ is an annulus. If $G_{P}$ has a Scharlemann cycle, then $\widehat{Q}$ must be separating in $M(\gamma)$.

(5) Any positive edge $\alpha$ of $\bar{G}_{Q}$ has $w(\alpha) \leq p / 2+1$. Moreover, if $w(\alpha)=p / 2+1$, then $\alpha$ contains an $S$-cycle.

(6) For any edge $\alpha$ of $\bar{G}_{Q}, w(\alpha)<p$.

(7) The edges of a Scharlemann cycle in $G_{P}$ cannot lie in a disk in $\widehat{Q}$.

Proof. (1) By the parity rule a positive level edge in $G_{Q}$ is a negative loop edge in $G_{P}$, which has a Möbius band neighborhood in $\widehat{P}$, contradicting the fact that $\widehat{P}$ is a sphere. (2) and (3) follow from [26, Lemma 5.2(2)-(3)]. (4) follows from [22, Lemma 2.2]. (5) By [26, Lemma 5.2(4)] $w(\alpha) \leq p / 2+1$. If $w(\alpha)=p / 2+1$, then $\alpha$ contains an $S$-cycle by [1, Corollary 2.6.7]. (6) is [22, Lemma $2.5(3)] .(7)$ is [22, Lemma 2.3].

Since $\widehat{P}$ is orientable, one can give a sign to each vertex of $G_{P}$ according to the sign of its intersection with the core of $V_{\pi}$. Two vertices (possibly equal) of $G_{P}$ are called parallel if they have the same sign, otherwise antiparallel. Similarly for the vertices of $G_{Q}$ when $\widehat{Q}$ is an annulus. A positive edge connects parallel vertices, while a negative one connects antiparallel vertices.

Lemma 5.5. Any cycle in $G_{P}$ contains an even number of negative edges.

Proof. A path through the edges of $G_{P}$ has the same sign at its end vertices if it contains an even number of negative edges, and the different signs otherwise. Hence the conclusion follows.

\section{Annulus}

Throughout this section we assume that $\widehat{Q}$ is an annulus. The purpose of this section is to prove that $G_{P}$ contains an $S$-cycle.

By Lemma $5.1 G_{Q}$ has exactly two vertices, $v_{1}$ and $v_{2}$. The reduced graph $\bar{G}_{Q}$ looks like Figure 7(a) (see the second paragraph in the proof of [26, Lemma 5.3]), so $G_{Q}$ has four edge classes, $\alpha, \beta, \varepsilon_{1}, \varepsilon_{2}$. Since $v_{1}$ and $v_{2}$ have the same valence, we have $w\left(\varepsilon_{1}\right)=w\left(\varepsilon_{2}\right)$. From now on, put $r=w\left(\varepsilon_{1}\right)=w\left(\varepsilon_{2}\right)$, and let $\varepsilon_{1}=\left\{e_{1}^{1}, e_{1}^{2}, \ldots, e_{1}^{r}\right\}$, $\varepsilon_{2}=\left\{e_{2}^{1}, e_{2}^{2}, \ldots, e_{2}^{r}\right\}$ as shown in Figure 7(b). We say that an edge in $G_{P}$ or $G_{Q}$ is an $\alpha$-edge (or a $\beta$-edge) if it belongs to class $\alpha$ (or $\beta$ ). By Lemma 5.4(6) we have $w(\alpha), w(\beta)<p$. Thus, $2 w\left(\varepsilon_{1}\right)=2 w\left(\varepsilon_{2}\right)=2 p-(w(\alpha)+w(\beta))$ implies $r>0$. From now on, we shall assume $w(\alpha) \geq w(\beta)$ and eventually show that the equality holds.

By Lemma 5.4(3), we may assume that if $G_{Q}$ contains Scharlemann cycles, then they have the same label pair, $\{1,2\}$.

Lemma 6.1. The two vertices of $G_{Q}$ are antiparallel.

Proof. Assume that $v_{1}$ and $v_{2}$ are parallel. Then all the edges of $G_{Q}$ are positive. For a label $x \neq 1,2$, consider the subgraph $\Gamma$ of $G_{Q}$ consisting of all vertices and all $x$-edges of $G_{Q}$. Let $V, E$ and $F$ be the numbers of vertices, edges and disk faces of $\Gamma$, respectively. Because of $V<E$, we have $0=\chi(\widehat{Q}) \leq V-E+F<F$, so $\Gamma$ contains a disk face, contradicting [19, Theorem 4.4].

Without loss of generality, we may assume that the ordering of labels around vertex $v_{1}$ is counterclockwise, while the ordering around vertex $v_{2}$ clockwise. All 


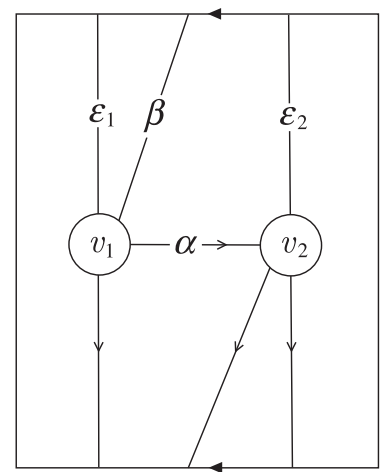

(a)

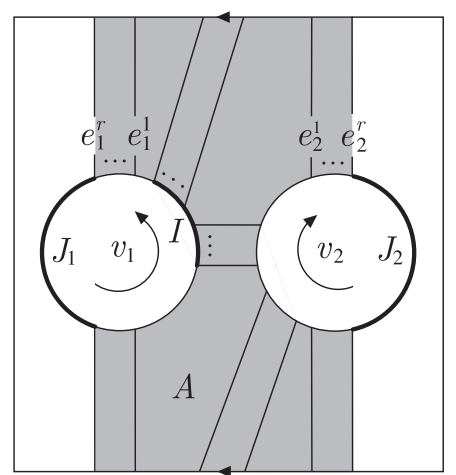

(b)

FiguRE 7.

of $\alpha$-edges and $\beta$-edges are negative in $G_{Q}$, and the others positive. By the parity rule, the set of the edges in $G_{P}^{+}$consists of the $\alpha$-edges and the $\beta$-edges, and any edge in $G_{P}^{+}$has label 1 at one endpoint and label 2 at the other.

Lemma 6.2. $G_{P}^{+}$contains disk faces.

Proof. We have $r \leq p / 2+1$ by Lemma 5.4(5). Assume $r<p / 2+1$. Then $r \leq(p+1) / 2$ and $G_{Q}$ contains at least $p-1$ negative edges, since $G_{Q}$ has $2 p$ edges. By the parity rule, $G_{P}^{+}$has at least $p-1$ edges. A usual Euler characteristic calculation for $G_{P}^{+}$shows that $G_{P}^{+}$contains a disk face.

Assume $r=p / 2+1$. By Lemma $5.4(3),(5) G_{Q}$ has two $S$-cycles with label pair $\{1,2\}$, which must be $\left\{e_{1}^{1}, e_{1}^{2}\right\}$ and $\left\{e_{2}^{1}, e_{2}^{2}\right\}$. Let $I$ be the shortest interval on $\partial v_{1}$ that contains all $\alpha$-edge-endpoints and all $\beta$-edge-endpoints around $v_{1}$. See Figure 7 (b). Labels $3,4, \ldots, p$ each appear exactly once on the interval $I$ because $e_{1}^{1}$ has label 2 at its south endpoint and label 1 at its north endpoint. The edge-endpoints contained in $I$ appear on $G_{P}$ as endpoints of positive edges with label 1 at vertices $u_{3}, u_{4}, \ldots, u_{p}$. That is, for any vertex $u_{j}$ other than $u_{1}$ and $u_{2}$, there is an edge of $G_{P}^{+}$incident to $u_{j}$ at some occurrence of label 1 . The edges $e_{1}^{1}, e_{1}^{2}, e_{2}^{1}, e_{2}^{2}$ together with the vertices $u_{1}, u_{2}$ divide the sphere $\widehat{P}$ into four disks. Choose one of these disks that contains some vertices of $G_{P}$ in its interior. Starting at a vertex of $G_{P}$ in the interior of the disk, one can construct a path through positive 1-edges. The path must hit the same vertex to create a 1-cycle. An innermost 1-cycle bounds a disk face of $G_{P}^{+}$.

For any disk face of $G_{P}^{+}$, its boundary is a Scharlemann cycle. Thus, Lemmas 5.4 (4) and 6.2 show that $\widehat{Q}$ divides $M(\gamma)$ into a black side $X$ and a white side $Y$. Also, $\partial \widehat{Q}$ divides $\partial M(\gamma)=\partial_{1} M$ into two annuli $B_{X}$ and $B_{Y}$ so that $\partial X=B_{X} \cup \widehat{Q}$ and $\partial Y=B_{Y} \cup \widehat{Q}$. Let $M_{X}=M \cap X, M_{Y}=M \cap Y$ and $H_{X}=V_{\gamma} \cap X, H_{Y}=V_{\gamma} \cap Y$. Let $A_{X}$ and $A_{Y}$ be the annuli $M_{X} \cap H_{X}$ and $M_{Y} \cap H_{Y}$. Then $\partial M_{X}=Q \cup A_{X} \cup B_{X}$ and $\partial M_{Y}=Q \cup A_{Y} \cup B_{Y}$ are genus 2 surfaces, and $Q=M_{X} \cap M_{Y}$. A face of $G_{P}$ is black or white according to whether it lies in $X$ or $Y$, and a corner of a face is black or white according to the color of the face. We may also think of a corner as a spanning arc in $A_{X}$ or $A_{Y}$. 


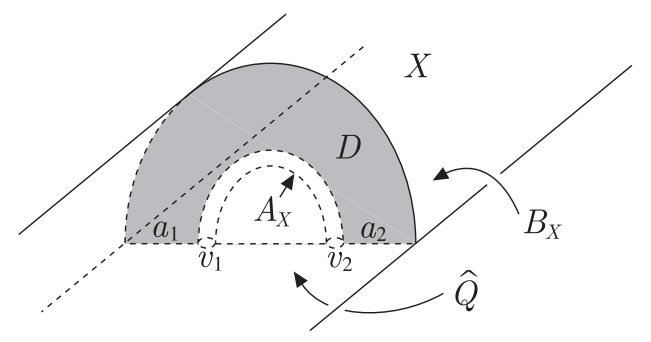

FiguRe 8.

Lemma 6.3. If $G_{P}^{+}$contains a disk face in $X$, then $M_{X}$ contains an essential disk $D$ such that

(i) $\partial D \cap A_{X}$ is a spanning arc in $A_{X}$;

(ii) $\partial D \cap B_{X}$ is a spanning arc in $B_{X}$; and

(iii) $\partial D \cap Q=a_{1} \cup a_{2}$, where $a_{i}$ is an essential arc in $Q$ disjoint from $P \cap Q$, and joins $\partial v_{i}$ to one component of $\partial \widehat{Q}(i=1,2)$. See Figure 8 .

The statement, with $X$ replaced by $Y$, also holds.

Proof. Let $f$ be a black disk face of $G_{P}^{+}$. Each edge in $\partial f$ is either an $\alpha$-edge or a $\beta$-edge. Let $u_{x_{1}}, u_{x_{2}}, \ldots, u_{x_{n}}$ be the vertices of $f$ and $\lambda_{j}$ the corner of $f$ at $u_{x_{j}}$, that is, the interval $f \cap u_{x_{j}}$ on $\partial u_{x_{j}}$. Each corner $\lambda_{j}$ is a spanning arc in $A_{X}$ and hence it has one endpoint at $\partial v_{1}$ and the other at $\partial v_{2}$. Let $J_{i}$ be an interval on $\partial v_{i}$ with $\partial J_{i}=\partial e_{i}^{r}$ whose interior contains no edge-endpoints of $G_{Q}(i=1,2)$. See Figure 7(b). Then $J_{i}$ is disjoint from the endpoints of $\left\{\lambda_{1}, \lambda_{2}, \ldots, \lambda_{n}\right\}$ at $\partial v_{i}$. Since the $\operatorname{arcs} \lambda_{1}, \lambda_{2}, \ldots, \lambda_{n}$ are parallel in $A_{X}$, one can take a rectangle $E$ on $A_{X}$ such that $E \cap \partial A_{X}=J_{1} \cup J_{2}, \partial E-\operatorname{Int}\left(J_{1} \cup J_{2}\right)$ is a union of two spanning $\operatorname{arcs}$ in $A_{X}$, and $E \cap\left(\lambda_{1} \cup \cdots \cup \lambda_{n}\right)=\emptyset$.

The edges $e_{1}^{r}$ and $e_{2}^{r}$ divide $Q$ into three annuli $A, A_{1}, A_{2}$, where $A_{i}$ is bounded by $e_{i}^{r} \cup J_{i}$ and one component of $\partial \widehat{Q}$. See Figure $7(\mathrm{~b})$. Then $A \cup A_{X}$ is a twicepunctured torus, on which $\partial f$ is a non-separating curve, since the vertices of $f$ are all parallel. Surgering $A \cup A_{X}$ along $f$ gives an annulus disjoint from the core of $V_{\gamma}$. But $E$ remains intact during the surgery, so it lies on this annulus. Slightly pushing the interior of this annulus into Int $X$ rel $E$ gives a properly embedded annulus $A_{0}$ in $X$, bounded by the two circles $e_{1}^{r} \cup J_{1}$ and $e_{2}^{r} \cup J_{2}$. Then $A^{\prime}=A_{0} \cup A_{1} \cup A_{2}$ is a properly embedded annulus in $M(\gamma)$, disjoint from the core of $V_{\gamma}$. Since $A^{\prime}$ has the same boundary as $\widehat{Q}$, it is incompressible in $M$. Thus $A^{\prime}$ must be parallel to $B_{X}$ in $M_{X}$, and hence we can take a disk $D$ in $M_{X}$ satisfying the conclusion of this lemma.

Observe that all disk faces of $G_{P}^{+}$are of the same color; if both sides $X$ and $Y$ contain disk faces of $G_{P}^{+}$, then $M_{X}$ and $M_{Y}$, respectively, contain essential disks $D_{X}$ and $D_{Y}$ satisfying the conclusion in Lemma 6.3. Isotope $D_{X}$ in $M_{X}$ so that $Q \cap D_{X}=Q \cap D_{Y}$. Then $D_{X} \cup D_{Y}$ becomes an annulus with one boundary component on $\partial_{0} M$ and the other on $\partial_{1} M$. Each boundary circle of this annulus is essential on the corresponding boundary component of $M$ by the properties (i) and (ii) in Lemma 6.3. Then either the annulus is an essential annulus in $M$ or it is compressible, giving a boundary reducing disk for $M$. Both contradict the fact that $M$ is simple. 
From now on, we shall assume that all disk faces of $G_{P}^{+}$are black.

Lemma 6.4. $G_{P}$ is connected.

Proof. Assume $G_{P}$ is disconnected. Then there exists a disk $E$ on $\widehat{P}$ such that $G_{E}=E \cap G_{P}$ and $G_{E^{\prime}}=(\widehat{P}-E) \cap G_{P}$ are both non-empty subgraphs of $G_{P}$. Put $k=\left|E \cap V_{\pi}\right|<p$. Let $G_{Q}^{E}$ be the subgraph of $G_{Q}$ consisting of all the vertices of $G_{Q}$ and the edges of $G_{Q}$ corresponding to the edges of $G_{E}$. As in Section 5, we can regard $G_{E}, G_{Q}^{E}$ as a pair of labelled graphs that arises from the intersection of $E$ and $Q$. We borrow the definitions and terminology of [13] and [4, in particular, the notions of a k-type, a k-interval and a great web. Then by [13, Theorem 3.3] either $G_{E}$ contains a great web or $G_{Q}^{E}$ represents all k-types. We show both are impossible.

Suppose that $G_{Q}^{E}$ represents all k-types. For each k-interval $\lambda=(i, i+1)$, we denote $H_{\lambda}$ the 1-handle consisting of that part of $V_{\pi}$ between the fat vertices numbered $i$ and $i+1$. For a set $\mathcal{D}$ of disk faces of $G_{Q}^{E}$, we define a 3-manifold $N(E, \mathcal{D})$ in $M(\pi)$ by

$$
N(E, \mathcal{D})=\operatorname{nbhd}\left(E \cup \bigcup_{\lambda \in c(\mathcal{D})} H_{\lambda} \cup \mathcal{D}\right),
$$

where $c(\mathcal{D})$ is the set of $\mathbf{k}$-intervals that occur as corners of elements in $\mathcal{D}$. Then by [4. Theorem 4.3] there exists a set $\mathcal{D}_{0}$ of disk faces of $G_{Q}^{E}$ such that $H_{1}\left(N\left(E, \mathcal{D}_{0}\right)\right)$ is finite and non-zero, and $\partial N\left(E, \mathcal{D}_{0}\right)$ is a sphere. However, $\partial N\left(E, \mathcal{D}_{0}\right)$ is a reducing sphere in $M(\pi)$ with $\left|\partial N\left(E, \mathcal{D}_{0}\right) \cap V_{\pi}\right|=\left|E \cap V_{\pi}\right|<p$, contradicting the minimality of $p$.

Suppose that $G_{E}$ contains a great web $\Lambda$. Notice that $\Lambda$ is a subgraph of $G_{P}^{+}$. By the definition of a great web, some vertex of $\Lambda$ must have at least 3 endpoints of edges in $\Lambda$. Then $\Lambda$ contains black and white disk faces simultaneously, contradicting the observation just above this lemma.

Orient all the vertices of $G_{P}$ (or $G_{Q}$ ) so that their boundaries are homologous on $\partial_{0} M$. Let $w$ be a vertex of $G_{P}$ (or $G_{Q}$ ). Let $A$ and $B$ be two edge-endpoints on $\partial w$. If $A=A_{0}, A_{1}, \ldots, A_{k}=B$ are the edge-endpoints encountered when travelling along $\partial w$ in the direction induced by the orientation of $w$, then we define the distance from $A$ to $B$ as $\tau_{P}(A, B)=k$ (or $\tau_{Q}(A, B)=k$ ).

We will repeatedly apply the following.

Lemma 6.5 ([5, Lemma 2.4(ii)]). Let $a, b$ be components of $\partial P$ and $x, y$ components of $\partial Q \cap \partial_{0} M$. Suppose that $A \in a \cap x, B \in a \cap y, C \in b \cap x$ and $D \in b \cap y$. If $\tau_{P}(A, B)=\tau_{P}(C, D)$, then $\tau_{Q}(A, C)=\tau_{Q}(B, D)$.

Orienting the edges in $G_{Q}$ according to the orientations of the edges in $\bar{G}_{Q}$ as shown in Figure 7(a), we may think of $G_{P}$ as a directed graph. Four edge-endpoints at each vertex of $G_{P}$ divides the boundary of the vertex into four corners, two black corners and two white corners. If an edge-endpoint at a vertex of $G_{P}$ belongs to an $\alpha$-edge or a $\beta$-edge, then it is called a positive edge-endpoint, otherwise a negative edge-endpoint.

Lemma 6.6. (1) For all $j=1,2, \ldots, r$, the edges $e_{1}^{j}$ and $e_{2}^{j}$ are parallel in $G_{P}$ with their heads (resp. tails) joined by a black corner.

(2) Each vertex of $G_{P}$ contains two or four positive edge-endpoints. 
(3) No black corner connects $\beta$-edge-endpoints.

(4) Each vertex $u_{x}$ of $G_{P}$ contains at most two $\alpha$-edge-endpoints (resp. $\beta$ edge-endpoints). Moreover, if $u_{x}$ contains two such points, then they are adjacent along $\partial u_{x}$.

Proof. (1) Since $G_{P}^{+}$has black disk faces, $M_{X}$ contains an essential disk satisfying the conclusion of Lemma 6.3. Among such disks, we choose a disk $D$ so that $D \cap P$ has a minimal number of components. Since $P$ is incompressible in $M$, a standard cut and paste argument shows that $D \cap P$ has no circle component. Any arc component of $D \cap P$, if it exists, must have both endpoints in the $\operatorname{arc} a=\partial D \cap A_{X}$. A subdisk of $D$ bounded by an outermost arc component of $D \cap P$ in $D$ along with a subarc on $a$ could be used to boundary compress $P$, contradicting Lemma 5.2 . Thus, $D \cap P=\emptyset$ and, in particular, all black corners are disjoint from the arc $a$, so parallel to $a$ in $A_{X}$. If $J_{i}$ is an interval on $\partial v_{i}$ as defined in the proof of Lemma $6.3(i=1,2)$, then the arc $a$ has one endpoint in each of $\operatorname{Int} J_{1}$ and $\operatorname{Int} J_{2}$. It follows that the heads (resp. tails) of $e_{1}^{j}$ and $e_{2}^{j}$ are joined by a black corner. By Lemma $6.4 e_{1}^{j}$ and $e_{2}^{j}$ must bound a bigon in $G_{P}$.

(2) Each vertex of $G_{P}$ contains an even number of positive edge-endpoints by (1). Thus it remains to show that there is no isolated vertex in $G_{P}^{+}$. To the contrary assume that a vertex $u_{x}$ of $G_{P}$ contains no positive edge-endpoints. Then $u_{x}$ is incident to two $\varepsilon_{1}$-edges and two $\varepsilon_{2}$-edges, and then in $G_{Q}$, label $x$ appears twice at the ends of $\varepsilon_{i}(i=1,2)$. By Lemma 5.4(6) and [1, Lemma 2.6.6], $\varepsilon_{i}$ contains an $S$-cycle, which must be $\left\{e_{i}^{1}, e_{i}^{2}\right\}$ by Lemma $5.4(2)$, since $\left\{e_{i}^{r-1}, e_{i}^{r}\right\}$ cannot form an $S$-cycle. The $S$-cycle $\left\{e_{i}^{1}, e_{i}^{2}\right\}$ has the label pair $\{1,2\}$ by our assumption. The subgraph of $G_{P}$ consisting of vertices $u_{1}, u_{2}$ and edges $e_{1}^{1}, e_{1}^{2}, e_{2}^{1}, e_{2}^{2}$ is a connected component of $G_{P}$, and in fact, all of $G_{P}$ by Lemma 6.4. This means $p=2$, contradicting the fact that $M$ is simple.

(3) We may assume that the boundaries of the vertices of $G_{P}$ are oriented so that all the black corners are oriented from the endpoint labelled 1 to the endpoint labelled 2. Assume that two $\beta$-edge-endpoints, $A$ and $B$, at a vertex $u_{x}$ are connected by a black corner in $\partial u_{x}$, where $A$ is labelled 1 and $B$ is labelled 2 . By (1), the heads of $e_{1}^{1}$ and $e_{2}^{1}, C$ and $D$, are connected by a black corner in the boundary of some vertex $u_{y}$. Since $\tau_{P}(A, B)=\tau_{P}(C, D)=1$, we have $\tau_{Q}(A, C)=\tau_{Q}(B, D)$ by Lemma 6.5. For each vertex $v_{i}(i=1,2)$ of $G_{Q}$, orient $\partial v_{i}$ according to the ordering of labels around $v_{i}$ so that $\partial v_{1}$ and $\partial v_{2}$ are homologous on $\partial_{0} M$. Then, by considering the locations of the points $A, B, C, D$ in $G_{Q}$, one can see that $\tau_{Q}(A, C) \leq w(\beta)$ and $\tau_{Q}(B, D) \geq w(\alpha)+1$. These inequalities give $w(\alpha)<w(\beta)$, contradicting our assumption $w(\alpha) \geq w(\beta)$ at the beginning of this section.

(4) Suppose that a vertex $u_{x}$ has three $\alpha$-edge-endpoints. Then two of them have label 1, say, and in $G_{Q}$, label $x$ appears twice in the set of $\alpha$-edge-endpoints at vertex $v_{1}$. This implies $w(\alpha)>p$, contradicting Lemma 5.4(6). Similarly, every vertex has at most two $\beta$-edges. The same argument also shows the second assertion.

For each $j=1,2, \ldots, r$, the edges $e_{1}^{j}$ and $e_{2}^{j}$ are parallel and bound a black bigon in $G_{P}$. We say that such a pair of edges is an $\varepsilon$-edge pair.

Lemma 6.7. Let $u_{x}$ and $u_{y}$ be the end vertices of a black bigon of $G_{P}$ bounded by an $\varepsilon$-edge pair. If $A$ is a positive edge-endpoint at $u_{x}$ with label 1 and $B$ is a positive edge-endpoint at $u_{y}$ with label 2 , then both $A$ and $B$ are either $\alpha$-edge-endpoints or $\beta$-edge-endpoints. 
Proof. Let $e_{1}^{j}$ and $e_{2}^{j}$ be the $\varepsilon$-edge pair, $j=1,2, \ldots, r$. By Lemma 6.6(1), the tails of $e_{1}^{j}$ and $e_{2}^{j}$ lie at the same vertex, say, at $u_{x}$. Let $C$ be the endpoint of $e_{2}^{j}$ at $u_{x}$, and $D$ the endpoint of $e_{1}^{j}$ at $u_{y}$. Since $u_{x}$ and $u_{y}$ are antiparallel, $\tau_{P}(A, C)=\tau_{P}(D, B)$. Lemma 6.5 leads to $\tau_{Q}(A, D)=\tau_{Q}(C, B)$. Since $C$ is the tail of $e_{2}^{j}$ and $D$ is the head of $e_{1}^{j}$, the result follows.

By Lemma 6.4 every face of $G_{P}$ is a disk. The graph $G_{P}$ is a directed graph on the 2 -sphere $\widehat{P}$. Thus, travelling around each face of $G_{P}$ gives a cyclic sequence of signed labels of the edges on the boundary of the face. As in Section 4 , we say that two faces of $G_{P}$ of the same color are isomorphic if one can obtain equal cyclic sequence of signed labels of the edges when travelling around the faces in some directions.

Recall that $\partial M_{X}=Q \cup A_{X} \cup B_{X}$ and $\partial M_{Y}=Q \cup A_{Y} \cup B_{Y}$ are genus two surfaces. The boundary of each face $f$ of $G_{P}$ lies on the twice-punctured torus $Q \cup A_{X}$ or $Q \cup A_{Y}$, according to its color, and each component of $\partial f \cap Q$ is an edge in $G_{Q}$. The circle $\partial f$ is essential in the twice-punctured torus; if $\partial f$ is null-homotopic in the surface, then $G_{Q}$ must have a 1-sided face, and if $\partial f$ is boundary parallel in the surface, then $f$ together with the boundary parallelism can be used to compress $B_{X}$ or $B_{Y}$, implying that $M$ is boundary-reducible. We say that $f$ is separating if $\partial f$ is separating in the twice-punctured torus, and non-separating otherwise. For example, if $f$ is a disk face of $G_{P}^{+}$, then it is a non-separating black face.

Neither side of $Q$ contains a pair of non-separating disk faces of $G_{P}$ which are not mutually parallel. Otherwise, (say) $M_{Y}$ cut along such a pair of disk faces would be a 3-ball, since $M$ is simple and then $B_{Y}$ would compress in this 3-ball, implying that $M$ is boundary-reducible. Therefore, each side of $Q$ contains at most two classes of non-isotopic disk faces, one separating and the other non-separating.

Lemma 6.8. Any two non-separating faces of $G_{P}$ of the same color are isomorphic, and so are any two separating faces of $G_{P}$ of the same color.

Proof. For example, let $f$ and $g$ be any two black disk faces of $G_{P}^{+}$. The circles $\partial f$ and $\partial g$ are parallel in $Q \cup A_{X}$ and hence there is an annulus $A$ in $Q \cup A_{X}$ with $\partial A=\partial f \cup \partial g$. Each component of $\partial A-\operatorname{Int} A_{X}$ is an edge of $G_{Q}$ in $\partial f$ or $\partial g$, while each component of $\partial A \cap A_{X}$ is a corner of $f$ or $g$. Note that $\partial A_{X}$ must intersect $A$ in spanning arcs of $A$, otherwise some edge of $G_{Q}$ in $\partial f$ or $\partial g$ would be a trivial loop in $G_{Q}$. Thus, $A-\operatorname{Int} A_{X}$ is a union of disjoint rectangles $R_{1}, \ldots, R_{n}$. Each $R_{i}$ realizes a parallelism between two edges $\partial f \cap R_{i}$ and $\partial g \cap R_{i}$. This completes the proof.

We remark that if a face of $G_{P}$ is a black bigon bounded by an $\varepsilon$-edge pair, then it is a separating black face.

Lemma 6.9. Any separating white face in $G_{P}$ has at least four positive edges whose orientations are not consistent along the boundary of the face.

Proof. Let $f$ be a separating white face of $G_{P}$. Note that $F=Q \cup A_{Y}$ is a twicepunctured torus such that $\partial f \cap A_{Y}$ is a non-empty set of spanning arcs in $A_{Y}$ and no component of $\partial f \cap Q$ is boundary parallel in $Q$. By Lemma 3.2, $\partial f \cap Q$ consists of edges $c_{1}, \ldots, c_{2 n}$ in $G_{Q}$ (successively labelled along $\partial f$ ) with the property that

(i) $c_{n}$ and $c_{2 n}$ are an $\varepsilon_{1}$-edge and an $\varepsilon_{2}$-edge; and 


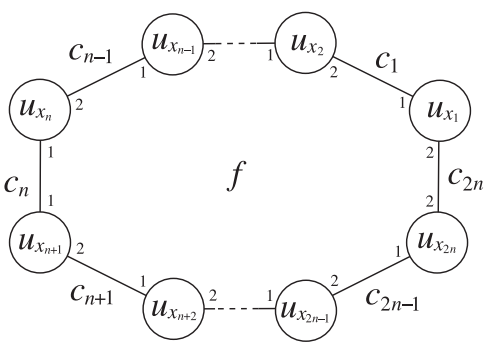

(a)

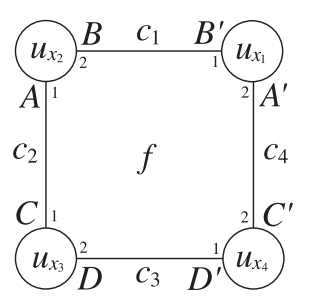

(b)

Figure 9.

(ii) there is a set of disjoint rectangles $\left\{R_{1}, \ldots, R_{n-1}\right\}$ on $Q$ such that $R_{j}$ realizes the parallelism between $c_{j}$ and $c_{2 n-j}$.

Thus, $c_{j}$ and $c_{2 n-j}$ belong to the same edge class. Let $\left\{u_{x_{1}}, \ldots, u_{x_{2 n}}\right\}$ be the vertices of $f$, which are labelled so that $u_{x_{j}}$ is incident to the edges $c_{j-1}$ and $c_{j}$ modulo $2 n$. See Figure 9 (a).

If $n=1$, then $c_{1}$ and $c_{2}$ are an $\varepsilon_{1}$-edge and an $\varepsilon_{2}$-edge which are parallel through the white bigon $f$. In $G_{Q}$, they have the same label pair, $\left\{x_{1}, x_{2}\right\}$, at their endpoints, so they form an $\varepsilon$-edge pair. By Lemma 6.6 (1) their endpoints are joined by black corners. However, their endpoints are also joined by white corners $f \cap \partial u_{x_{1}}$ and $f \cap \partial u_{x_{2}}$. This is impossible because $\Delta=2$.

If $n=2$, then $f$ is bounded by four edges $c_{1}, c_{2}, c_{3}$ and $c_{4}$. Let $A, B, C, D, A^{\prime}, B^{\prime}$, $C^{\prime}, D^{\prime}$ be their endpoints as illustrated in Figure 9(b). By (i), $u_{x_{1}}$ and $u_{x_{4}}$ are antiparallel, and so are $u_{x_{2}}$ and $u_{x_{3}}$. Thus, $\tau_{P}(A, B)=\tau_{P}(C, D)$ and $\tau_{P}\left(A^{\prime}, B^{\prime}\right)=$ $\tau_{P}\left(C^{\prime}, D^{\prime}\right)$. Lemma 6.5 leads to $\tau_{Q}(A, C)=\tau_{Q}(B, D)$ and $\tau_{Q}\left(A^{\prime}, C^{\prime}\right)=\tau_{Q}\left(B^{\prime}, D^{\prime}\right)$. By (ii), $\tau_{Q}(B, D)=\tau_{Q}\left(B^{\prime}, D^{\prime}\right)$ and hence $\tau_{Q}(A, C)=\tau_{Q}\left(A^{\prime}, C^{\prime}\right)$, which implies $\left(c_{2}, c_{4}\right)$ is an $\varepsilon$-edge pair. Then $u_{x_{1}}=u_{x_{2}}, u_{x_{3}}=u_{x_{4}}$, and $G_{P}$ consists of the two vertices $u_{x_{1}}, u_{x_{3}}$ and the four edges $c_{1}, c_{2}, c_{3}, c_{4}$ by Lemma 6.4. This contradicts the fact that $p \geq 3$. Therefore, $n>2$.

For any two consecutive edges $c_{j-1}$ and $c_{j}$, at least one of them must be positive, otherwise $u_{x_{j}}$ would have no positive edge-endpoint by Lemma 6.6 (1). This contradicts Lemma 6.6 (2). Thus the edges $c_{1}, c_{n-1}, c_{n+1}$ and $c_{2 n-1}$ are positive. The orientations of $c_{1}$ and $c_{2 n-1}$ (resp. those of $c_{n-1}$ and $c_{n+1}$ ) are opposite along $\partial f$, since $c_{1}$ and $c_{2 n-1}$ (resp. $c_{n-1}$ and $c_{n+1}$ ) belong to the same edge class by (ii). This completes the proof of the lemma.

Every non-separating black face of $G_{P}$ is a disk face of $G_{P}^{+}$, whose boundary is a Scharlemann cycle. Since all the $\alpha$-edges are oriented from the endpoint labelled 1 to the endpoint labelled 2 , while all the $\beta$-edges are reversely oriented, any black non-separating face of $G_{P}$ is not a cycle face by Lemma 5.4 (7). Every separating black face of $G_{P}$ is a black bigon bounded by an $\varepsilon$-edge pair, which is not a cycle face by Lemma 6.6(1). Lemma 6.9 shows that any separating white face of $G_{P}$ is also not a cycle face. Any vertex of $G_{P}$ is neither a sink nor a source; if $u_{x}$ is, for example, a source, then in Figure 7(a), label $x$ appears twice at the tails of $\alpha$ and $\varepsilon_{1}$, and twice at the tails of $\beta$ and $\varepsilon_{2}$, implying $w(\alpha)+w\left(\varepsilon_{1}\right)>p, w(\beta)+w\left(\varepsilon_{2}\right)>p$ and hence $w(\alpha)+w(\beta)+w\left(\varepsilon_{1}\right)+w\left(\varepsilon_{2}\right)>2 p$, which is impossible. Thus, every non-separating white face of $G_{P}$ must be a cycle face by [8, Lemma 2.3.1]. This 

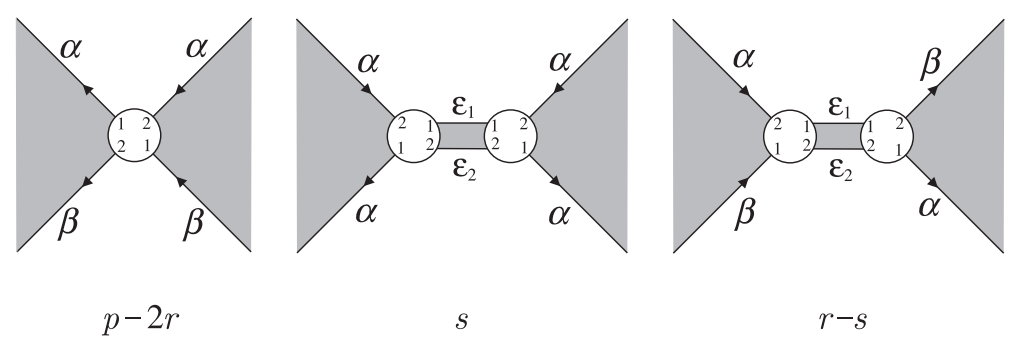

$r-s$

FigURE 10.

shows that the separating white faces and the non-separating white faces are not isomorphic.

There are $r \varepsilon$-edge pairs and hence $2 r$ end vertices of such pairs in $G_{P}$. So there are $p-2 r$ vertices of $G_{P}$ which have four positive edge-endpoints. Lemma 6.6 (3),(4) shows that the local view of $G_{P}$ near such a vertex looks like the first diagram in Figure 10. Lemmas 6.6(1),(2) and 6.7 show that the local view of $G_{P}$ near an $\varepsilon$-edge pair looks like one of the last two diagrams in Figure 10. Let $s$ be the number of $\varepsilon$-edge pairs whose end vertices have no $\beta$-edge-endpoint as in the second diagram.

Now we shall consider a new directed graph on the 2-sphere by ignoring the black bigons bounded by $\varepsilon$-edge pairs. Shrinking fat vertices of $G_{P}$ to points and then collapsing such black bigons, we obtain a new directed graph $\Gamma$ on the 2sphere whose local view near a vertex looks like one of the three diagrams in Figure 5. By Lemmas 6.8 and 6.9. $\Gamma$ satisfies the condition of Proposition 4.2 (the nonseparating black faces, non-separating white faces and separating white faces of $G_{P}$, respectively, correspond to the faces in classes $\mathcal{B}, \mathcal{C}$ and $\mathcal{A}$ in the proposition).

If the assertion (2) of Proposition 4.2 holds, then each non-separating black face of $G_{P}$ is bounded only by $\alpha$-edges, contradicting Lemma 5.4)(7). If the assertion (3) of Proposition 4.2 holds, then each non-separating white face of $G_{P}$ is bounded by the edges from the first diagram of Figure 10 and then it must be a disk face of $G_{P}^{+}$, contradicting our assumption that all disk faces of $G_{P}^{+}$are black. Therefore, we have $s=0$.

On the other hand, we wish to compute $w(\alpha)$ and $w(\beta)$ in terms of $p, r$ and $s$. The first, second and third diagrams in Figure 10 contribute 2, 4 and 2 to $2 w(\alpha)$, respectively. Hence $2 w(\alpha)=2(p-2 r)+4 s+2(r-s)$, giving $w(\alpha)=p-r+s$. Similarly, $w(\beta)=p-r-s$. Since $s=0$, we have $w(\alpha)=w(\beta)$.

Proposition 6.10. $M(\gamma)$ contains a properly embedded Möbius band.

Proof. We first show that $G_{P}$ contains an $S$-cycle. Orient $\partial v_{1}$ anticlockwise. Let $e$ and $e^{\prime}$ be the first $\alpha$-edge and the first $\beta$-edge in $G_{Q}$ that are encountered when going along the orientation of $\partial v_{1}$. By Lemma 6.6 $(1)$, the tails of $e_{1}^{1}$ and $e_{2}^{1}$ have the same label, say $x$ in $G_{Q}$. Then $e$ has a label pair, $\{x+1, x+w(\beta)+1\}$, at its endpoints, and $e^{\prime}$ has a label pair, $\{x+1, x+w(\alpha)+1\}$. Since $w(\alpha)=w(\beta)$, these label pairs are the same, and $\left\{e, e^{\prime}\right\}$ forms an $S$-cycle in $G_{P}$.

Let $f$ be a bigon in $G_{P}$ bounded by an $S$-cycle. Then $f$ must be black. By shrinking $H_{X}$ to its core, $H_{X} \cup f$ becomes a Möbius band $B$. The circle $\partial B$ is the core curve of the annulus $\widehat{Q}$, cutting it into two annuli. One of these annuli and $B$ produce a Möbius band properly embedded in $M(\gamma)$. 


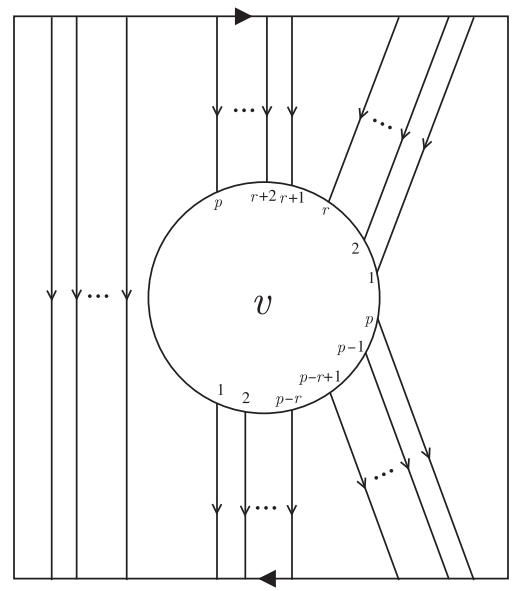

FiguRE 11.

\section{MÖBIUS BAND}

Throughout this section we assume that $\widehat{Q}$ is a properly embedded Möbius band in $M(\gamma)$. Let $\widehat{R}$ be the properly embedded annulus that is the frontier of a thin regular neighborhood of $\widehat{Q}$. Then $R=\widehat{R} \cap M$ is a twice-punctured annulus double covering $Q$. It is easy to see that $\widehat{R}$ is essential in $M(\gamma)$. We have defined a pair of labelled graphs $G_{P}$ and $G_{Q}$ in Section 5. As is done there, we obtain another pair of labelled graphs $G_{P}^{R}$ and $G_{R}$ from the intersection of $P$ and $R$. We can think of $G_{P}^{R}$ as the double of $G_{P}$.

By Lemma $5.1 G_{Q}$ has a single vertex $v$, so any edge of $G_{Q}$ is a loop edge. A Möbius band can contain at most two disjoint non-isotopic loops, one orientationpreserving and one orientation-reversing. Thus, the reduced graph $\bar{G}_{Q}$ has at most two edges. Up to relabelling, we may assume $G_{Q}$ is as shown in Figure 11 where $G_{Q}$ has $r$ positive edges and $p-r$ negative edges. The two families of parallel edges of $G_{Q}$ define a permutation on $p$ labels, $\{1,2, \ldots, p\}$, as follows. Orient the edges of $G_{Q}$ as shown in Figure 11 and define a permutation $\rho:\{1,2, \ldots, p\} \rightarrow\{1,2, \ldots, p\}$ so that $\rho$ carries the label at the tail of an edge onto the label at its head.

By Lemma 6.4 $G_{P}^{R}$ (and so also $G_{P}$ ) is connected. That is, $G_{P}$ becomes an embedded circle in $\widehat{P}$ after shrinking the fat vertices to points. This means that $\rho$ must be a cycle of length $p$, so $r=1$ or 2 by Lemma 3.3. If $r=1$, then $G_{P}$ has only one negative edge by the parity rule, contradicting Lemma 5.5. Therefore, $r=2$. Then $p$ must be even (and hence $p \geq 4$ ). This argument determines the pair of graphs $G_{Q}$ and $G_{P}$. We conclude that the integer $p$ determines the graphs $G_{R}$ and $G_{P}^{R}$ as illustrated in Figure 12 .

Proof of Theorem 1.1. The graph $G_{P}^{R}$ has two $p$-gons and $p$ bigons among which two bigons are bounded by negative edges and the others are bounded by positive edges. Let $f$ be a bigon bounded by positive edges and $g$ a $p$-gon, as indicated in Figure 12. Now we keep the notations and terminology in Section 6. Then $\partial f$ is an $S$-cycle consisting of one $\alpha$-edge and one $\beta$-edge. Thus, $f$ is a non-separating black face. The white face $g$ has one $\varepsilon_{1}$-edge and one $\varepsilon_{2}$-edge, which divide $\partial g$ into two parts. One part consists of only $\alpha$-edges and the other consists of only $\beta$-edges. 


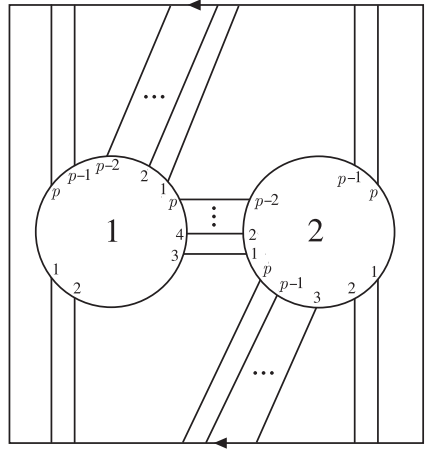

$G_{R}$

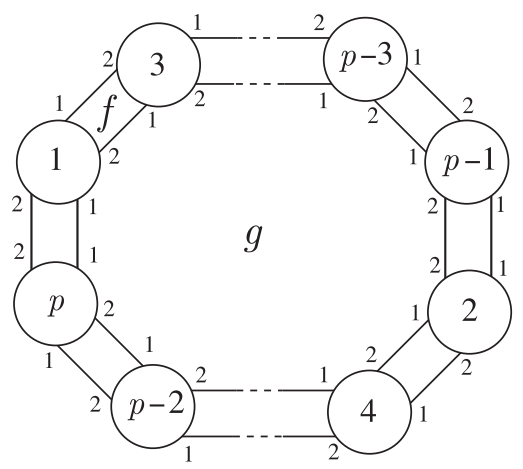

$G_{P}^{R}$

FiguRE 12.

Orienting the edges of $G_{R}$ according to Figure 7 (a), one can find a spanning arc in $\widehat{R}$ that intersects $\beta$-edges, $\varepsilon_{1}$-edges and $\varepsilon_{2}$-edges coherently, but does not intersect $\alpha$-edges. (For example, the bottom horizontal arc of the rectangle in Figure 12(a).) The edges of $g$ intersect this arc coherently in at least two points, so by Lemma 3.1, $g$ is non-separating.

Let $N$ be a regular neighborhood of $\partial_{0} M \cup Q \cup f \cup g$ in $M$. Surgering $Q \cup A_{X}$ along $f$ produces an annulus parallel to $B_{X}$, and similarly surgering $Q \cup A_{Y}$ along $g$ produces an annulus parallel to $B_{Y}$. Thus, $N$ is homeomorphic to $M$. This means that $M$ is uniquely determined by the pair of graphs $G_{R}$ and $G_{P}^{R}$, and hence by $p$.

Now consider $M(\gamma)$. One side of $\widehat{Q}, X$, is homeomorphic to a regular neighborhood of $\widehat{Q} \cup H_{X} \cup f$ and by [9, Lemma 3.7] it is a solid torus such that $\widehat{Q}$ runs twice longitudinally on its boundary. The other side, $Y$, is obtained from a genus two handlebody $\widehat{Q} \cup H_{Y}$ by attaching a 2-handle $g$. Calculating the fundamental group of the resulting space, one can verify that $Y=Q(p / 2+1,-p / 2-1)$ with $\widehat{Q} \subset \partial Y$ (see the proof of [2, Lemma 2.2.1]). Glue $X$ and $Y$ along $\widehat{Q}$ to get $M(\gamma)$. Since $\widehat{Q}$ runs twice longitudinally on the boundary of the solid torus $X$, the union of $X$ and a collar of $\partial Y$ in $Y$ produces a cable space $C(2,1)$. Thus, $M(\gamma)$ is the union of a cable space $C(2,1)$ and the Seifert fibered space $Q(p / 2+1,-p / 2-1)$ along their common torus boundary. So we conclude that $M$ is homeomorphic to $M_{p / 2+1}$ in Theorem 2.1.

\section{REFERENCES}

1. M. Culler, C. McA. Gordon, J. Luecke, and P.B. Shalen, Dehn surgery on knots, Ann. of Math. 125 (1987), 237-300. MR0881270 (88a:57026)

2. J. Dean, Small Seifert-fibered Dehn surgery on hyperbolic knots, Algebr. Geom. Topol. 3 (2003), 435-472. MR1997325 (2004m:57009)

3. M. Eudave-Muñoz and Y.Q. Wu, Nonhyperbolic Dehn fillings on hyperbolic 3-manifolds, Pacific J.Math. 190 (1999), 261-275. MR1722893 (2000j:57035)

4. C. McA. Gordon, Combinatorial Methods in Dehn surgery, Lectures at KNOTS '96 (Tokyo), Ser. Knots Everything, 15, World Sci. Publishing, River Edge, NJ, 1997, pp. 263-290. MR. 1474525 (98m:57020)

5. C. McA. Gordon, Boundary slopes on punctured tori in 3-manifolds, Trans. Amer. Math. Soc. 350 (1998), 1713-1790. MR1390037 (98h:57032) 
6. C. McA. Gordon, Small surfaces and Dehn fillngs, Geom. and Topol. Mono. 2 (1999), 177-199. MR $1734408(2000 \mathrm{j}: 57036)$

7. C. McA. Gordon and R. Litherland, Incompressible planar surfaces in 3-manifolds, Topology Appl. 18 (1984), 121-144. MR0769286 (86e:57013)

8. C. McA. Gordon and J. Luecke, Knots are determined by their complements, J. Amer. Math. Soc. 2 (1989), 371-415. MR0965210 (90a:57006a)

9. C. McA. Gordon and J. Luecke, Dehn surgeries on knots creating essential tori. I, Comm. Anal. Geom. 3 (1995), 597-644. MR.1371211 (96k:57003)

10. C. McA. Gordon and J. Luecke, Reducible manifolds and Dehn surgery, Topology 35 (1996), 385-409. MR 1380506 (97b:57013)

11. C. McA. Gordon and J. Luecke, Toroidal and boundary-reducing Dehn fillings, Topology Appl. 93 (1999), 77-90. MR1684214 (2000b:57030)

12. C. McA. Gordon and Y.Q. Wu, Toroidal and annular Dehn fillings, Proc. London. Math. Soc. 78 (1999), 662-700. MR1674841 (2000b:57029)

13. C. McA. Gordon and Y.Q. Wu, Annular and boundary reducing Dehn fillings, Topology 39 (2000), 531-548. MR.1746907(2001b:57033)

14. C. McA. Gordon and Y.Q. Wu, Annular Dehn fillings, Comment. Math. Helv. 75 (2000), 430-456. MR.1746907 (2001b:57033)

15. W. H. Jaco, and P. B. Shalen, Seifert fibered spaces in 3-manifolds, Mem. Amer. Math. Soc. 21 (1979), no. 220. MR0539411 (81c:57010)

16. K. Johannson, Homotopy equivalences of 3-manifolds with boundaries, Lecture Notes in Mathematics, 761, Springer, Berlin, (1979). MR0551744 (82c:57005)

17. H. Kneser, Geschlossene Flächen in dreidimensionale Mannigfaltigkeiten, Jahresber. Deutsch. Math.-Verein. 38 (1929) 248-260.

18. S. Lee, Reducing and toroidal Dehn fillings on 3-manifolds boundedy two tori, Math. Res. Lett. 13 (2006), 287-306.

19. S. Lee, S. Oh and M. Teragaito, Dehn fillings and small surfaces, Proc. London Math. Soc. 92 (2006), no. 1, 203-223. MR2192390

20. W.B.R. Lickorish, A representation of orientable combinatorial 3-manifolds, Ann. of Math. 76 (1962), 531-540. MR0151948 (27:1929)

21. J. Milnor, A unique decomposition theorem for 3-manifolds, Amer. J. Math. 84 (1962), 1-7. MR0142125 (25:5518)

22. R. Qiu, Reducible Dehn surgery and annular Dehn surgery, Pacific J. Math. 192 (2000), 357-368. MR 1744575 (2001b:57036)

23. W.P. Thurston, Three-dimensional manifolds, Kleinian groups and hyperbolic geometry, Bull. Amer. Math. Soc. 6(1982), 357-381. MR0648524 (83h:57019)

24. A. Wallace, Modifications and cobounding manifolds, Canad. J. Math. 12 (1960) 503-528. MR0125588 (23:A2887)

25. Y.Q. Wu, Dehn fillings producing reducible manifolds and toroidal manifolds, Topology $\mathbf{3 7}$ (1998), 95-108. MR1480879 (98j:57033)

26. Y.Q. Wu, Sutured manifold hierarchies, essential laminations, and Dehn surgery, J. Diff. Geom. 48 (1998), 407-437. MR 1638025 (99h:57043)

School of Mathematics, Korea Institute for Advanced Study, 207-43 CheongryangriDong, Dongdaemun-gu Seoul 130-722, Korea

E-mail address: slee@kias.re.kr

Current address: Department of Mathematics, Seoul National University, Seoul 151-747, Korea 